\title{
A HIGHER-ORDER GODUNOV METHOD FOR MULTIDIMENSIONAL IDEAL MAGNETOHYDRODYNAMICS ${ }^{*}$
}

\author{
ANDREW L. ZACHARY ${ }^{\dagger}$, ANDREA MALAGOLI ${ }^{\ddagger}$, AND PHILLIP COLELLA ${ }^{\S}$
}

\begin{abstract}
The authors present a higher-order Godunov method for the solution of the two- and three-dimensional equations of ideal magnetohydrodynamics (MHD). This work is based both on a suitable operator-split approximation to the full multidimensional equations, and on a one-dimensional Riemann solver. This Riemann solver is sufficiently robust to handle the nonstrictly hyperbolic nature of the MHD equations and the presence of local linear degeneracies. Results from a set of test problems show that this operator-split methodology has no problems handling any of the three MHD waves, yet resolves shocks to three or four computational zones. The advantages and limitations of this method are discussed.
\end{abstract}

Key words. magnetohydrodynamics (MHD), Godunov methods, hyperbolic systems, finite difference equations

AMS subject classifications. 65M06, 35L665, 3504, 76W05

1. Introduction. Conservative, finite-difference schemes based on higher-order Godunov methods have proven very effective at computing discontinuous solutions to hyperbolic systems of conservation laws. Several examples of such schemes are available for the equations of hydrodynamics (van Leer [23], Roe [20], Harten, Lax, and van Leer [13], and Colella and Woodward [9]) and have been used extensively to simulate highly supersonic flows in aerodynamics and astrophysics. For a comparative review of some of these methods, see, e.g., Woodward and Colella [24]. An extension of Godunov methods to general systems of hyperbolic conservation laws has been suggested by Bell, Colella, and Tragenstein [3], hereinafter refered to as BCT, who have also discussed modifications to the basic method when the systems have points at which they are no longer strictly hyperbolic. At these locations, some of the eigenvalues of the linearized matrix are identical and the corresponding eigenvectors become degenerate.

In this paper, we present a new, multidimensional scheme for the equations of ideal MHD, which is based on a higher-order Godunov method specially developed to treat the degeneracies that occur in these equations. Our formulation is a direct extension of the method developed by Zachary and Colella [25], but with several modifications and amendments that have proven necessary when testing the algorithm on a variety of one- and two-dimensional problems. The core of the problem is to construct an appropriate solution of a local, linearized Riemann problem along the ray $\frac{x}{t}=0$ at the boundary between two adjacent cell edges. This solution is then used to compute the flux $\mathbf{F}^{G}\left(\mathbf{U}_{R}, \mathbf{U}_{L}\right)$, the generalized Engquist-Osher [12] flux for our system of conservation laws (see BCT [3] for details).

Although this approach has several computational advantages over finding the solution to the complete nonlinear Riemann problem, it does have two major difficulties that require special attention. First, the equations of ideal MHD involve seven characteristics that can become mutually degenerate in several ways. Each type of degeneracy requires a different,

\footnotetext{
*Received by the editors June 22, 1992; accepted for publication (in revised form) June 24, 1993.

${ }^{\dagger}$ Cray Research, Inc., 397 Post Road, Darien, Connecticut 06820. The work of this author was supported by Cray Research Inc.

${ }^{\ddagger}$ Laboratory for Astrophysics and Space Research (LASR), University of Chicago, 933 E. 56th St., Chicago, Illinois 60637 (malagoli@mhd4.uchicago.edu). The work of this author was partly supported in part by National Aeronautics and Space Administration grant NAG 5-1485.

$\S$ Department of Mechanical Engineering, University of California, Berkeley, California 94720 (colella@barkley. Berkeley.edu). The work of this author was party supported at the University of California at Berkeley by Army Research Office grant DAALO3-88-K-0197. Defense Advanced Research Projects Agency and National Science Foundation grant DMS-8919074, and National Science Foundation Presidential Young Investigator award grant ACS-8958522.
} 
special treatment. Second, linearized Riemann solvers are known to fail in some extreme situations, even if the underlying physical problem admits a solution. Einfeldt et al. [11] have shown, for example, that certain types of hydrodynamic Riemann solvers fail to compute strong rarefaction waves correctly. Based on the experience obtained by computing a large set of test problems, we have incorporated an algorithm in our Riemann solver that detects and classifies the occurrence of degeneracies and strong rarefactions. This Riemann solver is so sufficiently robust that we have used it as the basis of a multidimensional scheme.

Following common procedures for higher-order methods, e.g., the piecewise parabolic method (PPM) and MUSCL schemes, the most natural way to extend our Riemann solver to two or three dimensions is by operator-splitting, in which a fully multidimensional step is achieved by combining a sequence of one-dimensional steps in alternating directions. However, the use of operator-splitting for the MHD equations cannot be achieved in a straightforward way, because the condition $\nabla \cdot \mathbf{B}=0$ introduces cross-coupling between the spatial directions. In this paper we have still adopted an operator-split scheme, because of its relative simplicity, and we have written the MHD equations in a particular nonconservation form that makes them suitable for operator-splitting. The condition $\nabla \cdot \mathbf{B}=0$ is further enforced by performing a projection (see, e.g., Brackbill and Barnes [4]) at the end of each timestep. A fully unsplit, multidimensional method will be developed at a later stage following the ideas of Colella [8].

In $\S 2$, we present an extensive description of the one-dimensional, higher-order Godunov method. We also briefly discuss our particular form of the operator-split MHD equations, and then we provide a detailed look at the Riemann solver. Finally, in $\S 3$, we present and discuss the results from our extensive series of one- and two-dimensional test problems.

2. The operator-split higher-order Godunov method. Zachary and Colella [25] describe a higher-order Godunov method designed for purely one-dimensional MHD equations. As with similar higher-order methods, e.g., PPM, MUSCL, BCT, this method has two parts: a predictor step that traces characteristics to determine time-centered values of the solution extrapolated to each cell edge from each of the left and right cell centers; and, a corrector step in which fluxes are computed at the cell edge and differenced to update the conserved quantities at the cell centers. These fluxes are computed using an approximate Riemann solver, using as input the left and right states obtained in the predictor step. In an earlier paper, we showed that our Riemann solver was sufficiently robust to handle the wave interactions present in a set of one-dimensional test problems. When these techniques are extended to a fully multidimensional set of MHD equations, they must handle a much wider range of wave phenomena. As a consequence, we have been led to make a number of modifications in our earlier solver to deal with these new interactions. Specifically, we must: account for terms of the form $\partial B_{x} / \partial x$ that arise from the operator-splitting; handle degeneracies that commonly arise in multidimensional studies, but which rarely occur in purely one-dimensional test problems; refine the treatment of rarefaction waves; and, finally, modify the predictor step to preserve monotonic gradients in both the characteristic fields and in the primitive variables. Since this solver differs significantly from the version described earlier, we present a detailed description of it below.

Before we begin our discussion, we make one general comment about our method. Many finite-difference algorithms such as MacCormack's method, Lax-Wendroff, and flux-corrected transport work only with $\mathbf{U}$, the conserved variables. Our experience with a higher-order Godunov method has led us to work with $\mathbf{U}$, as well as with the primitive variables $\mathbf{W}=$ $\left[\rho, u_{x}, u_{y}, u_{z}, B_{x}, B_{y}, B_{z}, p\right]^{t}$. There are several reasons for our choice. First, working in $\mathbf{W}$-space provides the simplest possible expressions for the right and left eigenvectors. Using these representations reduced the complexity and improved the performance of the resulting computer code. Second, our algorithm must preserve positivity in the density and the pressure. When the predictor or the corrector step used conserved variables, we found there was a broad 
range of conditions under which our Riemann solver produced a negative pressure. As our section below on rarefaction waves makes clear, switching to primitive variables fixed most, but not all, of the cases under which either the pressure or the density became negative.

2.1. Operator-split equations of MHD. The full set of equations for multidimensional hydrodynamics naturally splits into a set of one-dimensional equations with no cross-coupling. Unfortunately, this statement is not true for the equations of MHD; the restriction $\nabla \cdot \mathbf{B}=\mathbf{0}$ naturally induces some cross-coupling between the different directions. In this section, we present a set of one-dimensional equations for MHD that is suitable for operator-splitting. We begin with the full set of equations written as:

$$
\begin{aligned}
& \frac{\partial \rho}{\partial t}+\nabla \cdot(\rho \mathbf{u})=0 \\
& \frac{\partial \rho u_{x}}{\partial t}+\frac{\partial}{\partial x}\left(\rho u_{x}^{2}+p+\frac{B_{\perp . x}^{2}}{8 \pi}\right)+\nabla \cdot\left(\mathbf{u}_{\perp, x} u_{x} \rho\right)-\frac{1}{4 \pi}\left(\mathbf{B}_{\perp, x} \cdot \nabla\right) B_{x}=0 . \\
& \frac{\partial \rho u_{y}}{\partial t}+\frac{\partial}{\partial y}\left(\rho u_{y}^{2}+p+\frac{B_{\perp, y}^{2}}{8 \pi}\right)+\nabla \cdot\left(\mathbf{u}_{\perp, y} u_{y} \rho\right)-\frac{1}{4 \pi}\left(\mathbf{B}_{\perp, y} \cdot \nabla\right) B_{y}=0 . \\
& \frac{\partial \rho u_{z}}{\partial t}+\frac{\partial}{\partial z}\left(\rho u_{z}^{2}+p+\frac{B_{\perp, z}^{2}}{8 \pi}\right)+\nabla \cdot\left(\mathbf{u}_{\perp, z} u_{z} \rho\right)-\frac{1}{4 \pi}\left(\mathbf{B}_{\perp, z} \cdot \nabla\right) B_{z}=0, \\
& (1 \mathrm{f}) \frac{\partial(\rho E)}{\partial t}+\nabla \cdot(\rho \mathbf{u} E+\mathbf{u} p)+\sum_{i=1}^{3} \frac{\partial}{\partial x}\left(u_{x_{i}} \frac{B_{\perp . x_{i}}^{2}}{8 \pi}\right)-\frac{1}{4 \pi} \sum_{i=1}^{3} \frac{\partial}{\partial x_{i}} B_{x_{i}}\left(\mathbf{B}_{\perp . x_{i}} \cdot \mathbf{u}\right)=0 . \\
& \nabla \cdot \mathbf{B}=0 .
\end{aligned}
$$

The right-hand side of these equations differs from the conservation form of the equations by terms of the form $\nabla \cdot \mathbf{B}(\ldots)$, where the terms inside the parentheses are not differentiated. Our reason for using this form was to minimize the extent to which various terms in the discrete evolution operators in each of the coordinate directions in the operator split algorithm would have to sum to zero due to the divergence-free constraint on the magnetic field. The use of this nonconservation form is similar to the common practice, in incompressible flow calculations, of using advective differencing of the velocity fields, rather than conservative differencing (see, for example, Bell, Colella, and Glaz [1]). Also, it has been shown by Brackbill and Barnes [4] that using the nonconservation form of the momentum equations reduces the effect of magnetic monopole forces on the dynamics of the system. In the above equations, we have used the following notation:

$$
\begin{aligned}
\rho E & =\frac{1}{2} \rho u^{2}+\frac{p}{\gamma-1}+\frac{B^{2}}{8 \pi}, \\
\mathbf{B}_{\perp, x_{i}} & =\mathbf{B}-B_{x_{i}} \hat{\mathbf{e}}_{i}, \\
\mathbf{u}_{\perp, x_{i}} & =\mathbf{u}-u_{x_{i}} \hat{\mathbf{e}}_{i}, \\
\nabla \cdot\left(\mathbf{q}_{\perp, x_{i}} a\right) & =\sum_{i \neq j} \frac{\partial}{\partial x_{j}}\left(q_{x_{j}} a\right)=\nabla_{\perp, x_{i}}(\mathbf{q} a), \\
\mathbf{q}_{\perp, x_{i}} \cdot \nabla a & =\sum_{i \neq j} q_{j} \frac{\partial a}{\partial x_{j}}=\left(\mathbf{q} \cdot \nabla_{\perp, x_{i}}\right) a .
\end{aligned}
$$


With the equations written in this form, it is immediately apparent that a nonconservative, one-dimensional, operator-split set will be

$$
\begin{aligned}
& \frac{\partial \rho}{\partial t}+\frac{\partial \rho u_{x}}{\partial x}=0 \\
& \frac{\partial \rho u_{x}}{\partial t}+\frac{\partial}{\partial x}\left(\rho u_{x}^{2}+p+\frac{B_{\perp . x}^{2}}{8 \pi}\right)=0 . \\
& \frac{\partial \rho u_{y}}{\partial t}+\frac{\partial}{\partial x}\left(u_{x} u_{y} \rho\right)-\frac{B_{x}}{4 \pi} \frac{\partial B_{y}}{\partial x}=0, \\
& \frac{\partial \rho u_{z}}{\partial t}+\frac{\partial}{\partial x}\left(u_{x} u_{z} \rho\right)-\frac{B_{x}}{4 \pi} \frac{\partial B_{z}}{\partial x}=0, \\
& \frac{\partial B_{x}}{\partial t}=0, \\
& \frac{\partial B_{y}}{\partial t}+\frac{\partial}{\partial x}\left(u_{x} B_{y}-u_{y} B_{x}\right)=0 . \\
& \frac{\partial B_{z}}{\partial t}+\frac{\partial}{\partial x}\left(u_{x} B_{z}-u_{z} B_{x}\right)=0, \\
& \text { (2h) } \frac{\partial\left(\rho E^{1 \mathrm{D}}\right)}{\partial t}+\frac{\partial}{\partial x}\left(\rho u_{x} E^{1 \mathrm{D}}+u_{x} p+u_{x} \frac{B_{\perp . x}^{2}}{8 \pi}\right)-\frac{1}{4 \pi} \frac{\partial}{\partial x} B_{x}\left(B_{y} u_{y}+B_{z} u_{z}\right)=0 \text {. }
\end{aligned}
$$

Note that we have not completely eliminated terms of the form $\partial B_{x} / \partial x$; they appear in the $B_{y}, B_{z}$, and $\rho E$ evolution equations. However, since $\partial B_{x} / \partial t=0$, we can formally treat them as source terms.

The one-dimensional equations are all of the form

$$
\frac{\partial \mathbf{U}^{1 \mathrm{D}}}{\partial t}+\frac{\partial \mathbf{F}^{1 \mathrm{D}}}{\partial x}+\mathbf{A}\left(\mathbf{U}^{1 \mathrm{D}}\right) \frac{\partial \mathbf{H}\left(\mathbf{U}^{1 \mathrm{D}}\right)}{\partial x}=0
$$

where $\mathrm{U}^{1 \mathrm{D}}=\left[\rho, \rho u_{x}, \rho u_{y}, \rho u_{z}, B_{x}, B_{y}, B_{z}, \rho E^{1 \mathrm{D}}\right]^{T}$. On a one-dimensional row of cells, our discretization of these equations has the following form:

$$
\begin{aligned}
\mathbf{U}_{i}^{n+1}= & \mathbf{U}_{i}^{n}+\frac{\Delta t}{\Delta x}\left(\mathbf{F}\left(\mathbf{U}_{i-1 / 2}^{n+1 / 2}\right)-\mathbf{F}\left(\mathbf{U}_{i+1 / 2}^{n+1 / 2}\right)\right) \\
& +\frac{\Delta t}{\Delta x} \mathbf{A}\left(\frac{1}{2}\left(\mathbf{U}_{i+1 / 2}^{n+1 / 2}+\mathbf{U}_{i-1 / 2}^{n+1 / 2}\right)\right) \cdot\left(\mathbf{H}\left(\mathbf{U}_{i-1 / 2}^{n+1 / 2}\right) \mathbf{H}\left(\mathbf{U}_{i+1 / 2}^{n+1 / 2}\right)\right),
\end{aligned}
$$

where by $\mathrm{U}$ we mean $\mathrm{U}^{\mathrm{ID}}$. By adding the contribution of $B_{x}^{2}$ back into the energy, we arrive at an evolution operator for the conserved quantities, $U$. We denote this evolution operator by $\left(\mathbf{L}_{x}^{\Delta t} \mathbf{U}^{n}\right)_{i}-\mathbf{U}_{i}^{n+1}$. Similarly, we can define operators $\mathbf{L}_{y}^{\Delta t}$ and $\mathbf{L}_{z}^{\Delta t}$ for the one-dimensional equations in the $y$ and $z$ directions.

We can extend these one-dimensional operators to act on the full two- or three-dimensional grid of data,

$$
\begin{aligned}
& \left(\mathcal{L}_{x}^{\Delta t} \mathbf{U}\right)_{i, j, k}=\left(\mathbf{L}_{x}^{\Delta t} \mathbf{U}_{\cdot, j, k}\right)_{i}, \\
& \left(\mathcal{L}_{y}^{\Delta t} \mathbf{U}\right)_{i, j, k}=\left(\mathbf{L}_{y}^{\Delta t} \mathbf{U}_{i, \cdot k}\right)_{j}, \\
& \left(\mathcal{L}_{z}^{\Delta t} \mathbf{U}\right)_{i, j, k}=\left(\mathbf{L}_{z}^{\Delta t} \mathbf{U}_{i, j, \cdot}\right)_{k} .
\end{aligned}
$$


Our full operator-split scheme is given by

$$
\mathbf{U}_{i, j, k}^{n+1}=\left(\mathcal{L}_{x}^{\Delta t} \mathcal{L}_{y}^{\Delta t} \mathcal{L}_{z}^{\Delta t} \mathcal{L}_{z}^{\Delta t} \mathcal{L}_{y}^{\Delta t} \mathcal{L}_{x}^{\Delta t} \mathbf{U}^{n}\right)_{i, j, k},
$$

where we have used symmetric Strang splitting to obtain an overall evolution that is secondorder accurate in time provided the individual one-dimensional operators are second-order accurate.

2.2. The predictor step: tracing characteristics. As in other higher-order Godunov methods, we predict states at the zone interface by tracing characteristics. To trace characteristics, we must first construct an approximate gradient $\Delta \mathbf{W}$ for each primitive variable in each zone. There are two alternate ways to construct this gradient: construct $\Delta \mathbf{W}$ by either monotonizing each primitive variable or by monotonizing each characteristic field. In a purely hydrodynamic model, the monotonized central difference algorithm used in van Leer [23] and Colella [7] gives good results. This approximation, which we subsequently refer to as the MUSCL gradient, follows the first approach and monotonizes each primitive variable separately, perhaps with some nonlinear flattening algorithm. In a series of experiments with the MHD code on one-dimensional test problems, using this form of the gradient introduced moderate amplitude (5-10\%) fluctuations at shocks, compound waves, and certain types of rarefaction waves. These oscillations occur because under some conditions the MUSCL gradient is too steep. The usual cure-the flattening algorithm of [7]—when applied to the seven MHD variables, does not fix the problem.

The other approach, discussed in [3] and [8] for general systems of conservation laws, monotonizes each characteristic field separately. Unfortunately, this technique does not guarantee that the resulting gradient will be monotonic in each primitive variable. Rather than use either approach independently, we have adopted a modified hybrid of the two. This hybrid could be thought of as a MUSCL gradient combined with a highly sophisticated flattening algorithm.

Our method begins by computing eigenvalues, eigenvectors, and structure coefficients at each zone center. Zachary and Colella [25] give expressions for the eigenvalues and eigenvectors and we do not repeat them here. The structure coefficients, $\kappa_{l m}$, are the gradient of each wave speed in the direction of a characteristic field

$$
\kappa_{l m}=\left(\nabla \lambda_{l}\right) \cdot \mathbf{r}_{m} .
$$

We then form the following differences:

$$
\begin{aligned}
\Delta^{-} \mathbf{W} & =\left(\mathbf{W}_{i}-\mathbf{W}_{i-1}\right)=\sum \alpha_{k}^{-} \mathbf{r}_{k}, \\
\Delta^{+} \mathbf{W} & =\left(\mathbf{W}_{i+1}-\mathbf{W}_{i}\right)=\sum \alpha_{k}^{+} \mathbf{r}_{k}, \\
\Delta^{c} \mathbf{W} & =\frac{1}{2}\left(\mathbf{W}_{i+1}-\mathbf{W}_{i-1}\right)=\sum \alpha_{k}^{c} \mathbf{r}_{k}, \\
\Delta^{m} \mathbf{W} & =\sum \alpha_{k}^{m} \mathbf{r}_{k} .
\end{aligned}
$$

The expressions $\Delta^{-}, \Delta^{+}$, and $\Delta^{c}$ correspond to backward, forward, and centered differences, respectively. The final difference, $\Delta^{m}$, is the monotonized, fourth-order difference used in the MUSCL algorithm.

We combine these differences to construct a fourth-order monotonized gradient by monotonizing each characteristic field independently. That is, we write $\Delta \mathbf{W}$ as

$$
\Delta \mathbf{W}=\sum \bar{\alpha}_{k} \mathbf{r}_{k},
$$


where

$$
\bar{\alpha}_{k}= \begin{cases}\min \left(\gamma\left|\alpha_{k}^{-}\right|, \gamma\left|\alpha_{k}^{+}\right|,\left|\alpha_{k}^{c}\right|,\left|\alpha_{k}^{m}\right|\right) \times \operatorname{sign}\left(\alpha_{k}^{c}\right) & \text { if } \alpha_{k}^{-} \alpha_{k}^{+}>0, \\ 0 & \text { otherwise }\end{cases}
$$

The expansion coefficients $\alpha_{k}^{m}$ from the MUSCL gradient enforce monotonicity in each variable.

For strictly hyperbolic systems $\gamma=2$, however, if an eigenvector is locally linearly degenerate, we use $\gamma=1$. As in BCT, we detect local linear degeneracy through the structure coefficients. That is, at meshpoint $i$ and for a given characteristic $k$, whenever

$$
\kappa_{k k, i-1} \kappa_{k k, i}<0 \text { or } \kappa_{k k, i+1} \kappa_{k k, i}<0,
$$

we assume the corresponding eigenvector is linearly degenerate.

Using the monotonized expansion coefficients, we approximate the states at $\mathbf{W}_{i+1 / 2, L}^{n+1 / 2}$ and $\mathbf{W}_{i-1 / 2, R}^{n+1 / 2}$ by tracing each characteristic forward or backward from zone center. Following the discussion in $\mathrm{BCT}$, we write

$$
\begin{aligned}
& \mathbf{W}_{i+1 / 2, L}^{n+1 / 2}=\mathbf{W}_{i}^{n}+\frac{1}{2} \sum_{\lambda_{k}>0} \bar{\alpha}_{k}\left(1-\frac{\Delta x}{\Delta t} \lambda_{k}\right) \mathbf{r}_{k}, \\
& \mathbf{W}_{i-1 / 2, R}^{n+1 / 2}=\mathbf{W}_{i}^{n}-\frac{1}{2} \sum_{\lambda_{k}<0} \bar{\alpha}_{k}\left(1+\frac{\Delta x}{\Delta t} \lambda_{k}\right) \mathbf{r}_{k} .
\end{aligned}
$$

Finally, the predicted states, $\mathbf{W}_{L}$ and $\mathbf{W}_{R}$, are modified by the presence of source terms. These source terms can originate from: external forces, i.e., gravity; geometry, i.e., spherical or cylindrical coordinates; or $\partial B_{x} / \partial x$. Written in strict conservation form, the equations of MHD are each of the form

$$
\frac{\partial \mathbf{U}}{\partial t}+\nabla \cdot \mathbf{F}=S(\mathbf{U})
$$

where $S(\mathbf{U})$ represents sources terms. However, we have chosen to perform the characteristic analysis in Lagrangian or primitive variables, $\mathbf{W}$. In the $\mathbf{W}$-basis, (13) becomes

$$
\frac{\partial \mathbf{W}}{\partial t}+\mathbf{A}(\mathbf{W}) \cdot \frac{\partial \mathbf{W}}{\partial x}=S(\mathbf{W})
$$

The matrix $\mathbf{A}(\mathbf{W})$ is

$$
\mathbf{A}(\mathbf{W})=\left[\frac{\partial \mathbf{U}}{\partial \mathbf{W}}\right]^{-1} \frac{\partial \mathbf{F}}{\partial \mathbf{W}}
$$

Some straightforward algebra shows that the source terms must be transformed according to the rule

$$
S(\mathbf{W})=\left[\frac{\partial \mathbf{U}}{\partial \mathbf{W}}\right]^{-1} S(\mathbf{U})
$$

Assume for the moment that the only source terms come from the incorrect representation of $\partial B_{x} / \partial x$ in the $\mathbf{B}_{\perp}$ and in the $\rho E$ equations. Then we have

$$
S(\mathbf{U})=\left(0,0,0,0, u_{y} \frac{\partial B_{x}}{\partial x}, u_{z} \frac{\partial B_{x}}{\partial x}, \frac{\mathbf{u}_{\perp} \cdot \mathbf{B}_{\perp}}{4 \pi} \frac{\partial B_{x}}{\partial x}\right)^{T} .
$$


Using (15) to express $S(\mathbf{W})$ in terms of $S(\mathbf{U})$, we find that

$$
S(\mathbf{W})=\left(0,0,0,0, u_{y} \frac{\partial B_{x}}{\partial x}, u_{z} \frac{\partial B_{x}}{\partial x}, 0\right)^{T} .
$$

In the $\mathbf{W}$-representation, $\partial B_{x} / \partial x$ changes only the $\mathbf{B}_{\perp}$-equations, and not the pressure equation.

2.3. The corrector step. We seek solutions to the system of conservation laws

$$
\frac{\partial \mathbf{u}}{\partial t}+\nabla \cdot \mathbf{F}=\mathbf{0}
$$

As required by the Godunov methodology, we must compute the flux $\mathbf{F}^{G}\left(\mathbf{U}_{R}, \mathbf{U}_{L}\right)$ evaluated along the ray $\frac{x}{t}=0$ in the Riemann problem solution to the above equation with initial conditions

$$
\mathbf{U}(x, t=0)= \begin{cases}\mathbf{U}_{L} & x \leq 0, \\ \mathbf{U}_{R} & x>0 .\end{cases}
$$

As mentioned in [25], any general solution technique for the MHD version of (16) faces two serious difficulties. First, the MHD wave speeds are not strictly hyperbolic. The loss of strict hyperbolicity at a point means that the analytic structure of the weak solutions is generally unknown in the neighborhood of that point. Second, the genuinely nonlinear waves can be locally linearly degenerate. It is much more difficult to determine the correct entropy satisfying discontinuities for modes with local linear degeneracies.

To deal with these problems, the starting point for our methodology is a high-order Godunov method developed by BCT. We take the BCT point of view and treat the equations of MHD as a hyperbolic system of conservation laws whose weak solutions are uniquely determined by entropy conditions, such as those described in Liu [17] for these systems. The questions surrounding whether this general approach will produce the correct weak solutions are far from settled; some of these issues are discussed in BCT. The algorithm described by $\mathrm{BCT}$ is an extension of the Engquist-Osher [12] flux to general systems of conservation laws and is sufficiently robust to handle the nonstrictly hyperbolic nature of the MHD equations. In the exposition that follows, we rely on BCT for the higher-order solution of the Riemann problem.

As discussed in [25], we do not need the entire solution to the Riemann problem. Indeed, the full solution may not be well defined for nonstrictly hyperbolic systems, so it is sufficient to develop the solution as a series of approximations to the full Riemann problem along the ray $\frac{x}{t}=0$. In the next subsection, we describe the basic structure of our Riemann solver without any of the modifications needed to deal with rarefaction waves or with eigenvector degeneracies. As in the previous section on tracing characteristics, we continue to work in W-space.

2.3.1. The Riemann solver. At each interface, we expand the jump $\mathbf{W}_{R}-\mathbf{W}_{L}$ in terms of a linearly independent set of eigenvectors $\overline{\mathbf{R}}_{k}$, i.e.

$$
\mathbf{W}_{R}-\mathbf{W}_{L}=\sum_{k} \alpha_{k} \overline{\mathbf{R}}_{k}
$$

We determine $\overline{\mathbf{R}}_{k}$ from $\mathbf{W}_{e}=\frac{1}{2}\left(\mathbf{W}_{R}+\mathbf{W}_{L}\right)$, and let $\overline{\mathbf{R}}_{k}=\mathbf{r}_{k}\left(\mathbf{W}_{e}\right)$ be the $k$ th right eigenvector of $D \mathbf{F}$ at $\mathbf{W}_{e}$. 
With these expansion coefficients, we define two intermediate states that "bound" the entropy wave. These states are defined as

$$
\mathbf{W}_{L}^{*}=\mathbf{W}_{L}+\sum_{\lambda^{-}} \alpha_{k} \mathbf{r}_{k}, \quad \mathbf{W}_{R}^{*}=\mathbf{W}_{R}-\sum_{\lambda^{+}} \alpha_{k} \mathbf{r}_{k} .
$$

The $\lambda^{-}$waves move backward and the $\lambda^{+}$waves move forward with respect to the entropy wave.

At each of the four states $\mathbf{W}_{L}, \mathbf{W}_{L}^{*}, \mathbf{W}_{R}^{*}$, and $\mathbf{W}_{R}$, we compute eigenvalues for all seven MHD waves. From these eigenvalues, we can construct

$$
\bar{\lambda}_{0}^{*}=\frac{1}{2}\left(\lambda_{0 . L}^{*}+\lambda_{0, R}^{*}\right)
$$

which is the average advective velocity at the starred states. The sign of $\vec{\lambda}_{0}^{*}$ gives an unambiguous determination of the upwind state. If $\bar{\lambda}_{0}^{*} \geq 0$, then the upwind state lies to the left of the entropy wave, while if $\bar{\lambda}_{0}^{*}<0$, it lies to the right.

In our previous paper, we used the structure coefficients and the wave speeds at the leftand right-hand states and the two intermediate states to construct a cubic Hermite interpolating polynomial for each eigenvalue. We interpolated $\lambda^{-}$waves from $\lambda\left(\mathbf{W}_{L}\right)$ and $\lambda\left(\mathbf{W}_{L}^{*}\right)$ and $\lambda^{+}$. waves from $\lambda\left(\mathbf{W}_{R}\right)$ to $\lambda\left(\mathbf{W}_{R}^{*}\right)$. We used the resulting set of interpolants, $\bar{\lambda}_{k}$, to detect sonic points along each wave path.

In this study, we find that in the presence of large gradients, the structure coefficients can often become so large that they make the Hermite polynomial useless. These large structure coefficients occur when there are substantial changes in density and pressure between $\mathbf{W}_{e}$, the point at which we know the eigenvectors, and $\mathbf{W}_{L, R}$, or $\mathbf{W}_{L, R}^{*}$, where we compute $\nabla \lambda_{k}$. To circumvent this difficulty, we use a simple linear interpolation of the wave speeds from $\mathbf{W}_{L}$ to $\mathbf{W}_{L}^{*}$ and from $\mathbf{W}_{R}$ to $\mathbf{W}_{R}^{*}$. Changing to a linear, rather than a cubic, interpolation does not alter any of our results.

BCT derived a generalized version of the Engquist-Osher flux suitable for systems of nonstrictly hyperbolic equations. Ignoring for the moment the treatment of degenerate waves, and assuming that $\mathbf{U}_{L}$ is the upwind state, the $\mathrm{BCT}$ extension of the Engquist-Osher flux is

$$
\mathbf{F}^{E O}\left(\mathbf{U}_{L}, \mathbf{U}_{R}\right)=\mathbf{F}\left(\mathbf{U}_{L}\right)+\sum_{k=1}^{K}\left(\int_{0}^{\alpha_{k}} \min \left(\bar{\lambda}_{k}, 0\right) d \alpha\right) \cdot \overline{\mathbf{R}}_{k} .
$$

As BCT discuss, the integral terms in (20) add a nonnegative dissipation to each of the characteristic modes in the expansion of $\mathbf{U}_{R}-\mathbf{U}_{L}$.

Unfortunately, this generalization is unsuitable for multidimensional MHD because the terms of the form $\partial B_{x} / \partial x$ require special treatment, and because some terms in the flux must be differenced as gradients and some terms as divergences. We, therefore, adopt an alternative formulation of the Engquist-Osher flux. This alternative formulation was presented but not derived in Zachary and Colella [25]. We now discuss the origin of our flux formulation.

Our motivation is the scalar case, for which

$$
\begin{aligned}
F^{E O} & =f\left(u_{L}\right)+\int_{u_{L}}^{u_{R}} \min (a(u), 0) d u \\
& =f\left(u_{L}\right)+\int_{u_{L}}^{u_{R}} \chi(u) a(u) d u,
\end{aligned}
$$

where $a(u)$ is the wave speed along the path $u_{R}-u_{L}$, and $\chi(u)$ is the characteristic function 


$$
\chi(u)= \begin{cases}1 & \text { if } a(u)<0, \\ 0 & \text { if } a(u)>0 .\end{cases}
$$

This can be written as the sum of terms of the form

$$
\int_{u^{p}}^{u^{p+1}} a(u) d u=f\left(u^{P+1}\right)-f\left(u^{p}\right)
$$

where $a<0$ for all $u \in\left[u^{P}, u^{p+1}\right]$, and

$$
\begin{array}{rll}
a\left(u^{p}\right)=0 & \text { or } & u^{p}=u_{L}, \\
a\left(u^{p+1}\right)=0 & \text { or } & u^{p+1}=u_{R} .
\end{array}
$$

A simple formula for $F^{E O}$ is then given by

$$
F^{E O}=f\left(u_{L}\right)+\sum_{s=1}^{s}(-1)^{s} f\left(u^{s}\right),
$$

where $q=\operatorname{sign}\left(u_{R}-u_{L}\right), q u^{1}<q u^{2}<\cdots<q u^{S}$, and $u^{s}$ satisfies either $a\left(u^{s}\right)=0$ or

$$
\begin{array}{ll}
a\left(u^{1}\right)=0 & u^{1}=u_{L}, \\
a\left(u^{S}\right)=0 & u^{S}=u_{R} .
\end{array}
$$

We can use (24) as the starting point for systems. Given the approximate parameterization of the wave curves in $\S 2$ of BCT, we can define the Engquist-Osher flux for systems of equations as follows:

$$
\mathbf{F}^{E O}=\mathbf{F}\left(\mathbf{W}_{L}\right)+\sum_{k=1}^{K} \sum_{s=1}^{S(k)} \mathbf{F}\left(\mathbf{W}_{k}^{s}\right)(-1)^{s}
$$

where

$$
\begin{aligned}
& \mathbf{W}_{k}=\mathbf{W}_{L}+\sum_{k^{\prime}<k} \bar{\alpha}_{k^{\prime}} \mathbf{R}_{k^{\prime}}, \\
& \mathbf{W}_{k}^{s}=\mathbf{W}_{k}+\boldsymbol{\alpha}_{k}^{s} \mathbf{R}_{k} .
\end{aligned}
$$

Also, $q \alpha_{k}^{1}<q \alpha_{k}^{2}<\cdots<q \alpha_{k}^{S}$, and $q=\operatorname{sign}\left(\bar{\alpha}_{k}\right)$. Finally, $\alpha_{k}^{s}$ satisfies

$$
\begin{array}{rl}
\bar{\lambda}_{k}\left(\alpha_{k}^{s}\right)=0 & 2 \leq s \leq S(k)-1, \\
\bar{\lambda}_{k}\left(\alpha_{k}^{1}\right) \leq 0 & \alpha_{k}^{1}=0, \\
\bar{\lambda}_{k}\left(\alpha_{k}^{S(k)}\right) \leq 0 & \alpha_{k}^{S(k)}=\bar{\alpha}_{k},
\end{array}
$$

and $\bar{\lambda}_{k}(\alpha)$ is the linear approximation to the wave speed given above.

The advantage of this approach is that various subpieces of the flux can be defined, as might be required for quasi-conservative differencing. If $\mathbf{F}=\mathbf{Q}_{1}+\mathbf{Q}_{2}$, then $\mathbf{Q}_{i}$ can be defined as

$$
\mathbf{Q}_{i}^{E O}=\mathbf{Q}_{i}\left(\mathbf{W}_{l}\right)+\sum_{k=1}^{K} \sum_{s=0}^{S} \mathbf{Q}_{i}\left(\mathbf{W}_{k}\right)(-1)^{s} .
$$

Then $\mathbf{Q}_{1}$ could be differenced as a divergence, while $\mathbf{Q}_{2}$ could be differenced as a gradient, as might be required in a spatially varying geometry. 
2.3.2. Rarefaction waves. Rarefaction waves cause problems for most Riemann solvers. Even the fully nonlinear Riemann solver in PPM needs special modification to deal with rarefaction waves, and most other Riemann solvers simply treat rarefaction waves as if they were rarefaction shocks. Our technique of expanding the discontinuity at each zone interface in terms of eigenvectors at a single reference state also does not correctly handle rarefaction waves. In fact, Einfeldt et al. [11] show that there exist initial conditions for strong rarefaction waves under which any linear Riemann solver must fail. With this failure in mind, we have modified our Riemann solver whenever we detect a strong rarefaction. Our detection criterion is simply that if

$$
v_{f}\left(\mathbf{W}_{e}\right)<\lambda_{0}\left(\mathbf{W}_{R}\right)-\lambda_{0}\left(\mathbf{W}_{L}\right) .
$$

where $v_{f}$ is the velocity of the fast magnetosonic wave, then we assume there is a strong rarefaction wave at this interface. Using the procedures in Einfeldt et al., particularly, equations (4.4) and (4.5), we recompute the flux as

$$
\mathbf{F}^{G}\left(\mathbf{W}_{R}, \mathbf{W}_{L}\right)=\frac{b^{+} \mathbf{F}\left(\mathbf{W}_{R}\right)-b^{-} \mathbf{F}\left(\mathbf{W}_{L}\right)}{b^{+}-b^{-}}+\frac{b^{+} \cdot b^{-}}{b^{+}-b^{-}}\left(\mathbf{U}_{R}-\mathbf{U}_{L}\right) .
$$

Here, $b^{+}=\max \left(\lambda_{R}^{+}, \lambda_{e}^{+}, 0\right)$ and $b^{-}=\min \left(\lambda_{L}^{-}, \lambda_{e}^{-}, 0\right)$; see [11] for details. This particular representation of the Godunov flux is positively conservative.

Rarefaction waves also pose problems for our algorithm since we use density rather than specific volume as an independent variable. (Using specific volume rather than density poses similar problems at compressions.) These problems arise when estimating the density at intermediate states, particularly at $\mathbf{W}_{L, R}^{*}$. When we construct $\mathbf{W}_{L}^{*}$ or $\mathbf{W}_{R}^{*}$, if

$$
\rho\left(\mathbf{W}_{L}^{*}\right)<\rho\left(\mathbf{W}_{L}\right) \quad \text { or } \quad \rho\left(\mathbf{W}_{R}^{*}\right)<\rho\left(\mathbf{W}_{R}\right),
$$

then we interpolate in specific volume rather than in density on that particular side of the entropy wave. Using the eigenvectors in [25], we note that a change in basis from $\rho$ to $\tau$ only changes the density components of the right and left eigenvectors. The only nonzero density component of a left eigenvector belongs to the entropy wave, and since the left eigenvectors determine the expansion coefficients, only the expansion coefficient for the entropy wave, $\alpha_{0}$, changes under this change in basis. Note, however, that $\alpha_{0}$ is never used by construction. We simply keep track of the appropriate representation for the right eigenvectors on each side of the entropy wave. The rest of our method for constructing the Engquist-Osher flux remains unchanged.

2.3.3. Degenerate waves. The MHD equations are nonstrictly hyperbolic because there are points with degenerate eigenvalues. At these points, the wave ordering that applies in strictly hyperbolic systems no longer holds and two or more wave speeds coincide. In MHD, the eigenvalues become degenerate in two distinct limits: if $B_{x}=0$ and if $\mathbf{B}_{\perp}=0$. In the first limit, the Alfven and the slow magnetosonic wave travel at the same speed as the entropy wave, resulting in a five-fold degeneracy. In the second limit, there are three different degeneracies that can occur depending upon the values of the Alfven velocity, $v_{a x}$, and the sound velocity $c_{s}$. When $v_{a x}>c_{s}$, the fast magnetosonic and the Alfven wave are degenerate, while when $v_{a x}<c_{s}$, the Alfven wave and the slow magnetosonic wave are degenerate, and finally when $v_{a x}=c_{s}$, all three waves are mutually degenerate. In each limit, and for each subcase, the algorithm must detect any degeneracy and then take appropriate action.

It is important here to clarify the exact meaning of such degeneracies and why they are peculiar to MHD. According to the definition given by Lax [15], two or more waves are considered to be degenerate when their values coincide, resulting in a loss of strict hyperbolicity. 
In this case it may happen that the eigenvectors corresponding to the orginally distinct eigenvalues become essentially parallel, resulting in an eigenvector deficiency like those considered in BCT. Also, Lax [16] defines a wave to be linearly degenerate when $\left(\nabla \lambda_{k}\right) \cdot \mathbf{r}_{k}=0$, where $\mathbf{r}_{k}$ is the right eigenvector corresponding to the $k$ th characteristic field. This is the case, for example, for the entropy and Alfven waves.

We emphasize that with our choice of eigenvectors (Zachary and Colella [25]), the degeneracy of the eigenvalues does not also imply a deficiency in the eigenvectors. We always have a set of complete, linearly independent eigenvectors spanning the phase space. The same situation already occurs in the equations for pure three-dimensional hydrodynamics: the entropy wave has in fact a three-fold degeneracy that is related to the transport of entropy and shear, and there are three linearly independent eigenvectors associated with it. In pure hydrodynamics, the three-fold degeneracy always occurs, and is independent of the value of the physical variables. Therefore, purely hydrodynamics equations can still be viewed as a strictly hyperbolic system, and it can be shown that the Engquist-Osher formulation [12] does compute fluxes that satisfy the entropy condition. In MHD, however, waves that are genuinely nonlinear in the sense of Lax [16] (specifically, the fast and slow magnetosonic waves) do become linearly degenerate when their eigenvalues coincide with those of already linearly degenerate waves, i.e., the entropy and Alfven waves. If this condition happens anywhere along the wave-path of integration between the left and right states, then the Engquist-Osher formulation may fail and the entropy condition may not be satisfied. When this happens, we need to modify our scheme and apply a different type of dissipation, as described below.

The first task in dealing with degeneracies is detecting them. Analytically, two eigenvectors are degenerate whenever their corresponding eigenvalues coincide. It is rare, however, that eigenvalues will, numerically, be exactly equal; therefore we have adopted here the BCT detection algorithm. The BCT algorithm was designed with the idea in mind to identify points in a general system of hyperbolic conservation laws at which eigenvectors become parallel and the corresponding expansion coefficients are no longer reliable. However, the same approach can be utilized more generally to detect loss of strict hyperbolicty, independent of the fate of the eigenvectors. As already mentioned, our construction of the MHD eigenvectors insures that when the eigenvalues coincide, the corresponding eigenvectors are not deficient, but an originally genuinely nonlinear wave may become linearly degenerate. We adopt the BCT idea that two eigenvectors are degenerate whenever the difference in their eigenvalues at $\mathbf{W}_{e}$ is less than some fraction of the difference in the predicted variation of the eigenvalues over the wave path. Specifically, we say that two eigenvectors are degenerate whenever

$$
\left|\lambda_{l}^{e}-\lambda_{m}^{e}\right| \leq c \sum_{k}\left|\alpha_{k}\left(\kappa_{k l}-\kappa_{k m}\right)\right|
$$

(Here, as elsewhere, we use $c=0.1$.)

The eigenvector degeneracy detection criterion in (31) fails in the limit $B_{x} / \mathbf{B}_{\perp} \rightarrow 0$, where the Alfven and slow magnetosonic waves become degenerate, but where the structure coefficients are independent of $B_{x}$. (We define $\kappa_{l m}=\left(\nabla \lambda_{l}\right) \cdot \mathbf{r}_{m}$, but there is no component of $\mathbf{r}_{m}$ along $B_{x}$.) We, therefore, adopt an altemative criterion, empirically derived, which states that the eigenvectors corresponding to the Alfven and slow magnetosonic waves become degenerate whenever

$$
\frac{\left|v_{a x}^{e}\right|}{v_{f}^{e}} \leq c^{2} \frac{\left(\mathbf{B}_{\perp}^{2}\left(\mathbf{W}_{e}\right)+\mathbf{B}_{\perp}^{2}\left(\mathbf{W}_{L}\right)+\mathbf{B}_{\perp}^{2}\left(\mathbf{W}_{R}\right)\right)^{1 / 2}}{\left|B_{x}\left(W_{e}\right)\right|},
$$

where $v_{a x}^{e}$ and $v_{f}^{e}$ are, respectively, the Alfven velocity and fast mode velocity at $\mathbf{W}_{e}$. 
The linear Riemann solver described in the previous sections breaks the full Riemann problem into two distinct parts, with each part corresponding to waves that move forward or backward with respect to the entropy wave. Accordingly, we treat all waves that are degenerate with the entropy wave as if they were additional entropy waves. Recall that from (19), entropy waves are not included in the construction of $\mathbf{W}_{L . R}^{*}$, nor are they incorporated in the computation of the flux.

Any other changes in the flux computation depend upon whether the eigenvector degeneracy is associated with a change in the sign of the wave speed along the wave path. Assume that the eigenvalues $\lambda_{I}^{e}$ and $\lambda_{m}^{e}$ satisfy (31), and assume that $\bar{\lambda}_{0}^{*}>0$, so that the reference state is $\mathbf{W}_{L}$. Let

$$
\lambda_{l m}^{\max }=\max \left(\lambda_{l}^{L}, \lambda_{l}^{L^{*}}, \lambda_{m}^{L}, \lambda_{m}^{L^{*}}\right)
$$

and

$$
\lambda_{l m}^{\min }=\min \left(\lambda_{l}^{L}, \lambda_{l}^{L^{*}}, \lambda_{m}^{L}, \lambda_{m}^{L^{*}}\right) .
$$

When $\lambda^{\min }$ and $\lambda^{\max }$ have the same sign, (25) remains unchanged. When $\lambda_{l m}^{\min }$ and $\lambda_{l m}^{\max }$ have opposite signs, then we assume that the eigenvector degeneracy is associated with a transonic wave. In this case, we replace the flux correction terms in (25) with a dissipative term from Rusanov's [21] scheme. More exactly, the added flux correction term is

$$
\mathbf{F}^{E O}=\mathbf{F}\left(\mathbf{W}_{L}\right)-\frac{1}{2} \nu \sum_{k=l, m} \alpha_{k} \overline{\mathbf{R}}_{k}+\sum_{k \neq l, m} \sum_{s=1}^{S(k)} \mathbf{F}\left(\mathbf{W}_{k}^{s}\right)(-1)^{s},
$$

where $v=\max \left(\left|\lambda_{l m}^{\max }\right|,\left|\lambda_{l m}^{\min }\right|\right)$. Although this description of a Rusanov-type dissipation assumes that only two waves were degenerate, it is easily generalized to include the case where many different waves are degenerate.

Note that if (32) is true, and the Alfven and slow waves are degenerate, then it is possible for waves to be degenerate across the entropy wave. In that case, the definitions for $\lambda^{\text {min }}$ and $\lambda^{\max }$ simply look at the wavespeeds at $\mathbf{W}_{L}$ and $\mathbf{W}_{R}$, without considering the wavespeeds at the intermediate states.

2.4. Insuring $\nabla \cdot \mathbf{B}=0$. The problem of preserving $\nabla \cdot \mathbf{B}=0$ to the highest possible accuracy, possibly even to machine accuracy, is a crucial one for discretized versions of the MHD equations. While the continuum equations insure that an initially solenoidal magnetic field will remain solenoidal when evolving in time, the discretized equations do not. As a consequence, numerical solutions of the MHD equations can generate magnetic monopole forces that strongly affect the dynamics of the fluid (See, e.g., Brackbill and Barnes [4]). Writing the equations in variables other than the magnetic field, like the vector potential or the magnetic flux function, partially solves the problem. This approach, however, has two drawbacks: it requires a staggered mesh, and the equations are not in a form suitable for characteristic analysis.

In our code we have used a centered, finite-difference scheme based on primitive variables because this scheme is the natural choice for higher-order Godunov methods. Our choice means that $\nabla \cdot \mathbf{B}=0$ is preserved only to the truncation error of the method. In principle, that truncation error could be $O(1)$, since we are computing solutions in the presence of discontinuities. To deal with that problem, we follow the prescription in [4], remove the nonsolenoidal part of $\mathbf{B}$, and then apply a Hodge projection to $\mathbf{B}$ at the end of each timestep. First solve the Poisson equation for the potential $\phi$

$$
\nabla^{2} \phi+\nabla \mathbf{B}=0,
$$


then the new solenoidal magnetic field is defined by $\mathbf{B}=\mathbf{B}-\nabla \phi$. Here we used second-order central difference approximations for the gradient and divergence operators. This numerical form of the Hodge projection leads to nonstandard discretizations of the Laplacian. However, the resulting linear equations can be solved efficiently and accurately using interative methods, e.g., multigrid (see Bell, Colella, and Howell [2]). It is worth noting that, for the two-dimensional problems presented in this paper, we did not find any noticeable difference regardless of whether or not we applied a projection.

3. Numerical results. We have tested our algorithm on a variety of MHD problems in one and two dimensions to check the consistency and robustness of our method under different conditions on the physical variables. Each problem tests different features of the code, such as its behavior in special limiting conditions, e.g., in the purely hydrodynamic limit, or its ability to track discontinuities. Our testing procedure has been particularly useful as we tuned the degeneracy detection algorithm, which is a crucial component of our Riemann solver.

All computations described below were performed on a Cray Y-MP8e/8128-4 at the Cray Research, Inc., data center in Eagan, Minnesota, and on a Cray Y-MP8/464 at the NASA Center for Computational Sciences (NCCS) in Greenbelt, Maryland. At the moment, the current version of the code, including all the test and corrections for degeneracy, the construction of two different sets of eigenvectors for each meshpoint, and the modifications for rarefaction waves, takes $15 \mu$ s per cell in one dimension and twice that in two dimensions. In other words, the algorithm does approximately 32,000 meshpoints/s in two dimensions. Our timestep was set by the Courant-Friedrichs-Lewy (CFL) stability condition

$$
\Delta t=\sigma \max _{i, j}\left(\frac{\Delta x_{i}}{\left|u_{x, i}\right|+v_{f, i}}, \frac{\Delta y_{i}}{\left|u_{y, j}\right|+v_{f, j}}\right),
$$

where $\sigma$ is the Courant number, and the maximum is taken over $i=1, \ldots, N_{x}, j=1, \ldots, N_{y}$. All the calculations were done using a Courant number $\sigma=0.8$ without any artificial viscosity.

3.1. One-dimensional problems. Our suite of one-dimensional problems includes benchmarks commonly used to test numerical algorithms: the Sod shock tube problem [22], the Brio and Wu problem [5], and the strong version of the Sod shock tube problem [5]. In addition, we have studied the strong rarefaction problems considered by Einfeldt et al. [11]. Each problem tests a different aspect of the Riemann solver. The Sod shock tube problem tests the solver in the purely hydrodynamic limit, the Brio and Wu problem stresses our handling of linear degeneracies and compound waves, while the strong version of the Brio and $\mathrm{Wu}$ problem accents our handling of large-amplitude shock waves. Finally, the Einfeldt et al. problems test our ability to detect and handle strong rarefaction waves.

As mentioned above, the Sod shock tube is a purely hydrodynamical Riemann problem in which the initial condition consists of two uniform states $\mathbf{W}_{L}$ and $\mathbf{W}_{R}$ separated by a discontinuity. The gas polytropic index is $\gamma=1.4$, and the initial conditions are

$$
\mathbf{W}_{L}(x<0)=\left[\begin{array}{c}
1 \\
0 \\
0 \\
0 \\
0 \\
0 \\
1
\end{array}\right], \quad \mathbf{W}_{R}(x>0)=\left[\begin{array}{c}
.125 \\
0 \\
0 \\
0 \\
0 \\
0 \\
0.1
\end{array}\right] .
$$


The numerical solution has been obtained for 100 grid points (with $\Delta x=1$ ) and is shown in Fig. 1 after 50 timesteps ( $\Delta t$ being given by the CFL condition). The shock discontinuity is well resolved within three grid points. The contact discontinuity is slightly more diffused, which is expected since we have not used a detection algorithm (see [9]). Also, there is a small undershoot at the base of the rarefaction wave that is a peculiar feature of how our linearized solver treats rarefaction waves. This undershoot can be partially eliminated by adding a quadratic artificial viscosity to our scheme of the type described by BCT. In Fig. 2 we show a comparison between the density profile at the same timestep as computed by our method and by the PPM method of Colella and Woodward [9]. Both solutions are very similar, with the main difference being the undershoot discussed above; one can see that the contact discontinuity is better represented by the PPM code that used a contact detection algorithm.

In Fig. 3 we present the results of the Brio and Wu [5] problem, which is an MHD analogue of the Sod shock tube problem. The initial configuration is the same as the Sod shock tube problem, but with $\gamma=2$; the initial magnetic field is $B y_{l}=\sqrt{4 \pi}, B y_{r}=-\sqrt{4 \pi}$, and $B x=0.75 \sqrt{4 \pi}$. Here we have used a grid of 800 points and 400 timesteps. The solution is in excellent agreement with the one obtained by Brio and Wu [5] using a Riemann solver written specifically for this problem. Again, we note that our method introduces a small perturbation at rarefaction waves. This perturbation does not affect the solution seriously and was previously noted also by Zachary and Colella [25]. On the other hand, all the shocks are extremely sharp and do not show any post-shock oscillations. In Fig. 4 we present the last of our set of Riemann problems, the strong version of the magnetized Sod shock tube problem that was also discussed by [5]. Here we set $B_{x}=0, P_{L}=1000, \gamma=\frac{5}{3}$, and all the other parameters as before. The solution is very well behaved even in this extreme case, and we note that we could solve this problem with a $\gamma \neq 2$, while the Brio and Wu solver could not.

Our final one-dimensional test problem is drawn from the strong rarefaction problems discussed by Einfeldt et al. [11]. In our earlier discussion of the Riemann solver, we noted that conditions exist under which any linear Riemann will fail, even if the underlying physical problem admits a solution. We have implemented a strong rarefaction wave detection algorithm that automatically applies additional dissipation whenever the Riemann solver fails. In Fig. 5 we show the results for the problem defined as $1-2-0-3$ in [11]. This problem consists of two strong rarefaction waves moving symmetrically in opposite directions. With an adiabatic index $y=1.4$, the initial conditions are

$$
\mathbf{W}_{L}(x<0)=\left[\begin{array}{c}
1 \\
-2 \\
0 \\
0 \\
0 \\
0 \\
0.4
\end{array}\right], \quad \mathbf{W}_{R}(x>0)=\left[\begin{array}{c}
l \\
2 \\
0 \\
0 \\
0 \\
0 \\
0.4
\end{array}\right] .
$$

From the analysis in [11], these initial conditions have a physical solution, but the solution is not linearizable.

3.2. Two-dimensional problems. Among the various two-dimensional test cases that we have tried, we present here two particularly interesting ones: a spherically symmetric explosion in a uniformly magnetized medium and the compressible version of the OrszagTang vortex (see Dahlburg and Picone [10] and Picone and Dahlburg [19]). Both problems develop interesting wave patterns and shocks that propagate in all directions, and therefore 

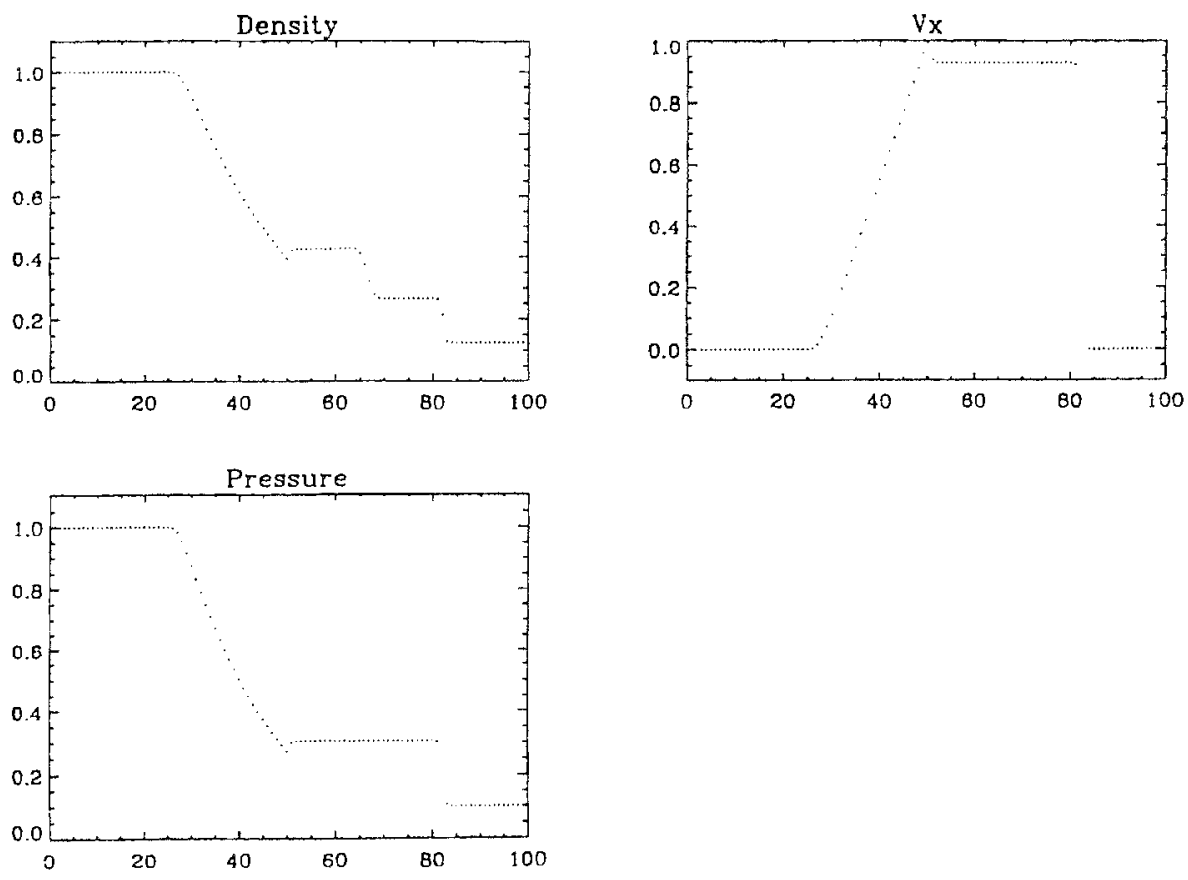

FIG. 1. The solution to the Sod shock tube problem on a uniform mesh with 100 grid points is shown after 50 timesteps. The size of the timestep was controlled by the CFL condition with CFL number of 0.8 .

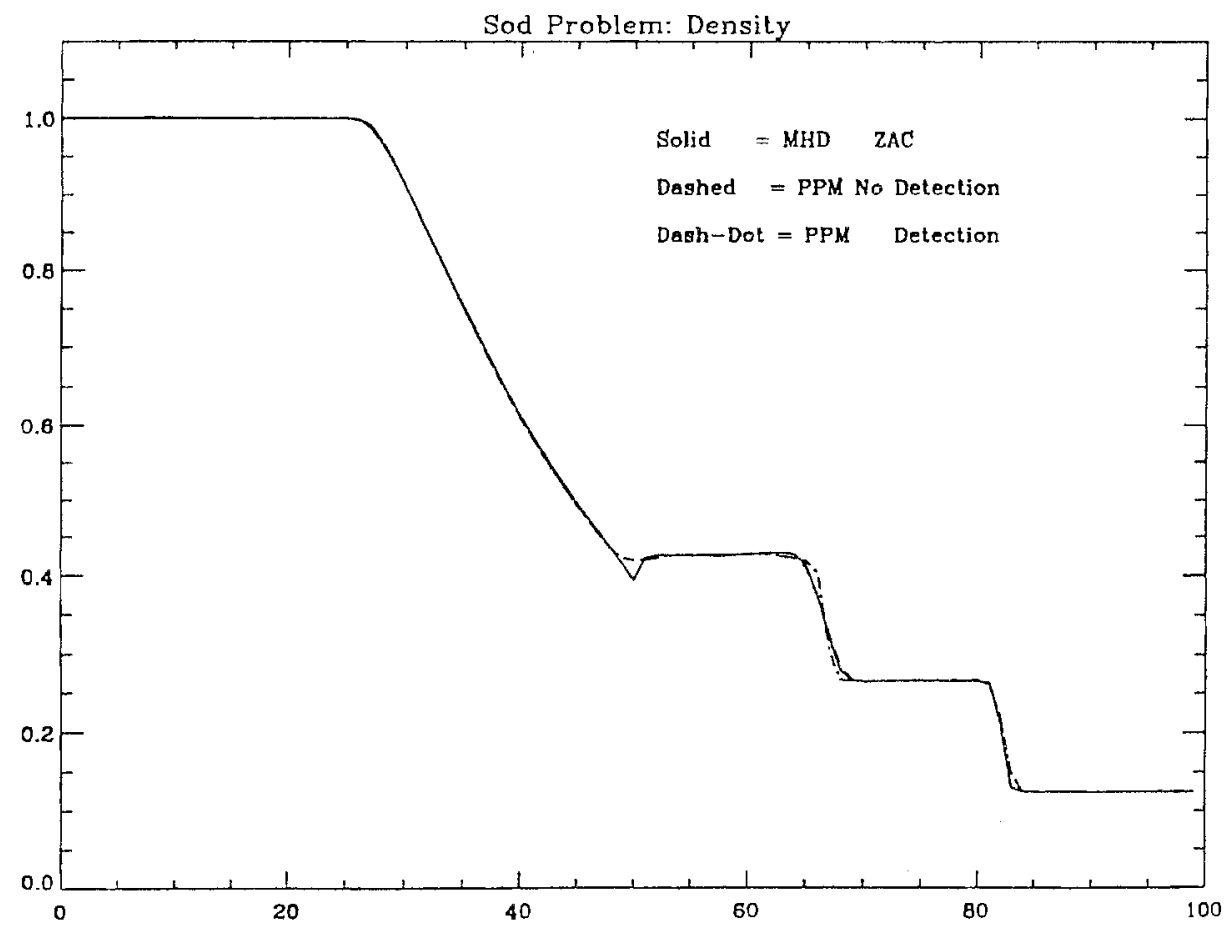

Fig. 2. The comparison between the density profiles of the solution to the Sod shock tube problem computed after 50 timesteps by using different algorithms is presented. The solid line is the solution computed with the present code; the dashed line is the solution computed with the PPM code in which the contact detection algorithm has been turned off; the dashed-dotted line is the PPM solution with detection of contact discontinuities. The effect of contact detection on the resolution of the contact discontinuity is clearly visible. 

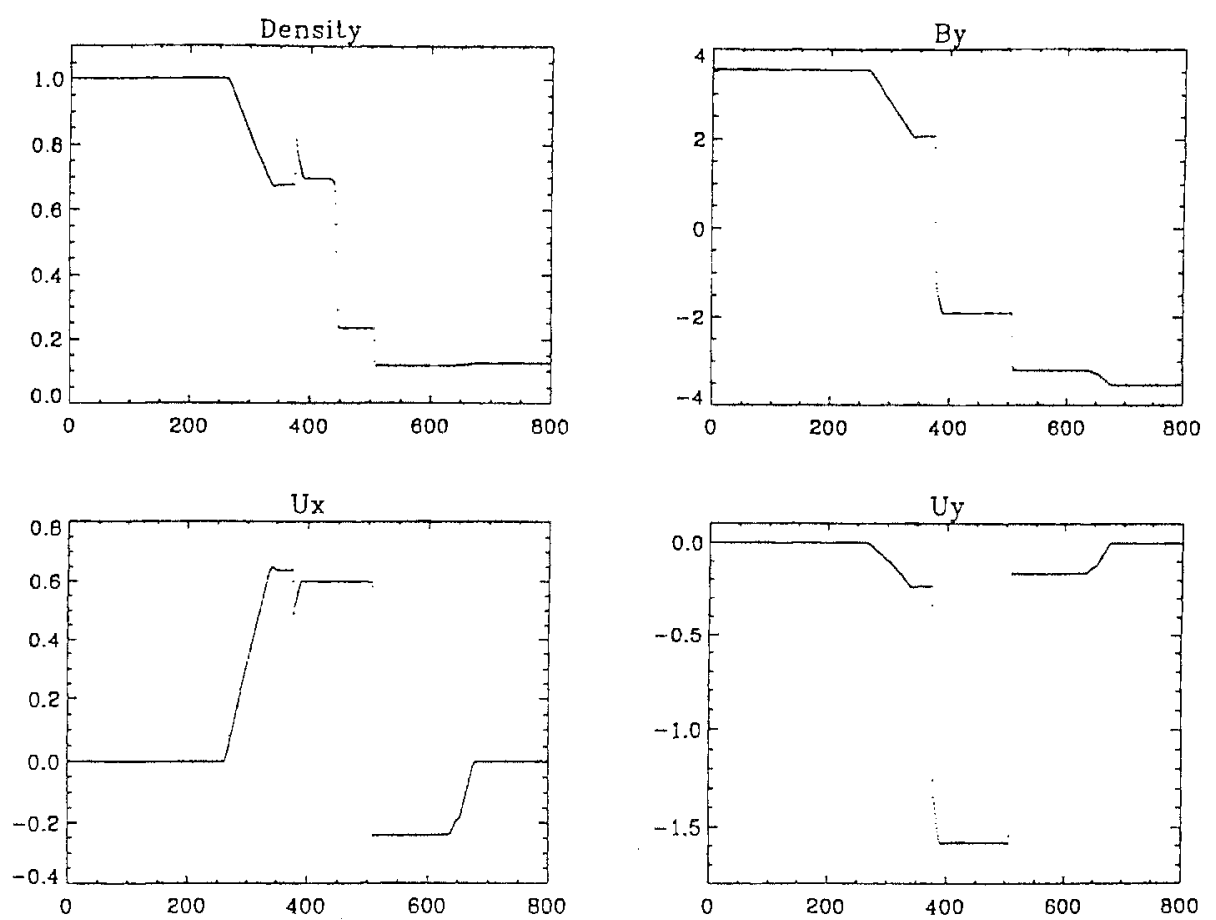

FIG. 3. The solution to the Brio and Wu magnetized shock tube problem on a uniform mesh with 800 grid points is shown after 400 timesteps. The initial condition consists of a jump discontinuity in pressure and density separated by a current sheet.
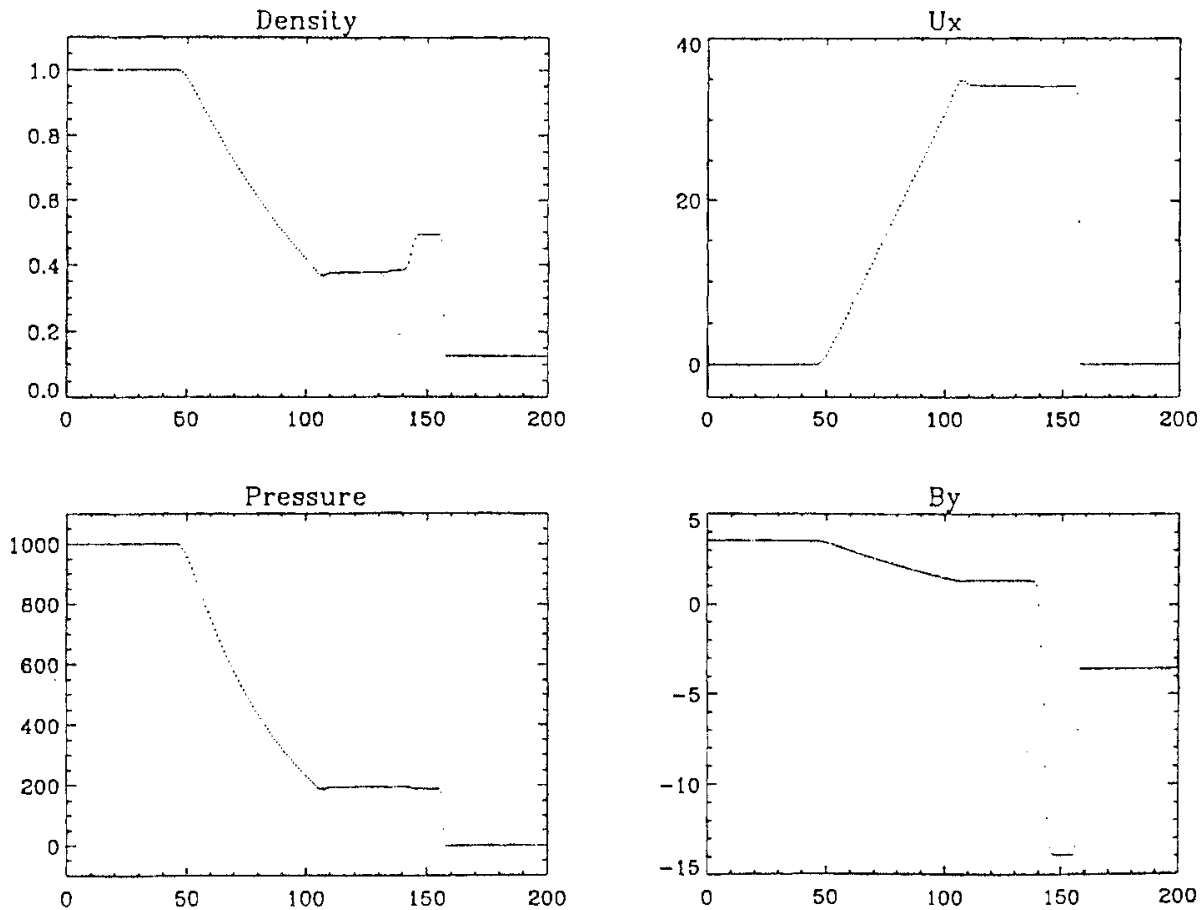

FIG. 4. The solution to the strong version of the shock tube problem on a mesh with 200 grid points is shown after 100 timesteps. Here $B_{x}=0$ and $B_{y}$ changes sign across the initial discontinuity. A CFL condition of 0.8 was used. 

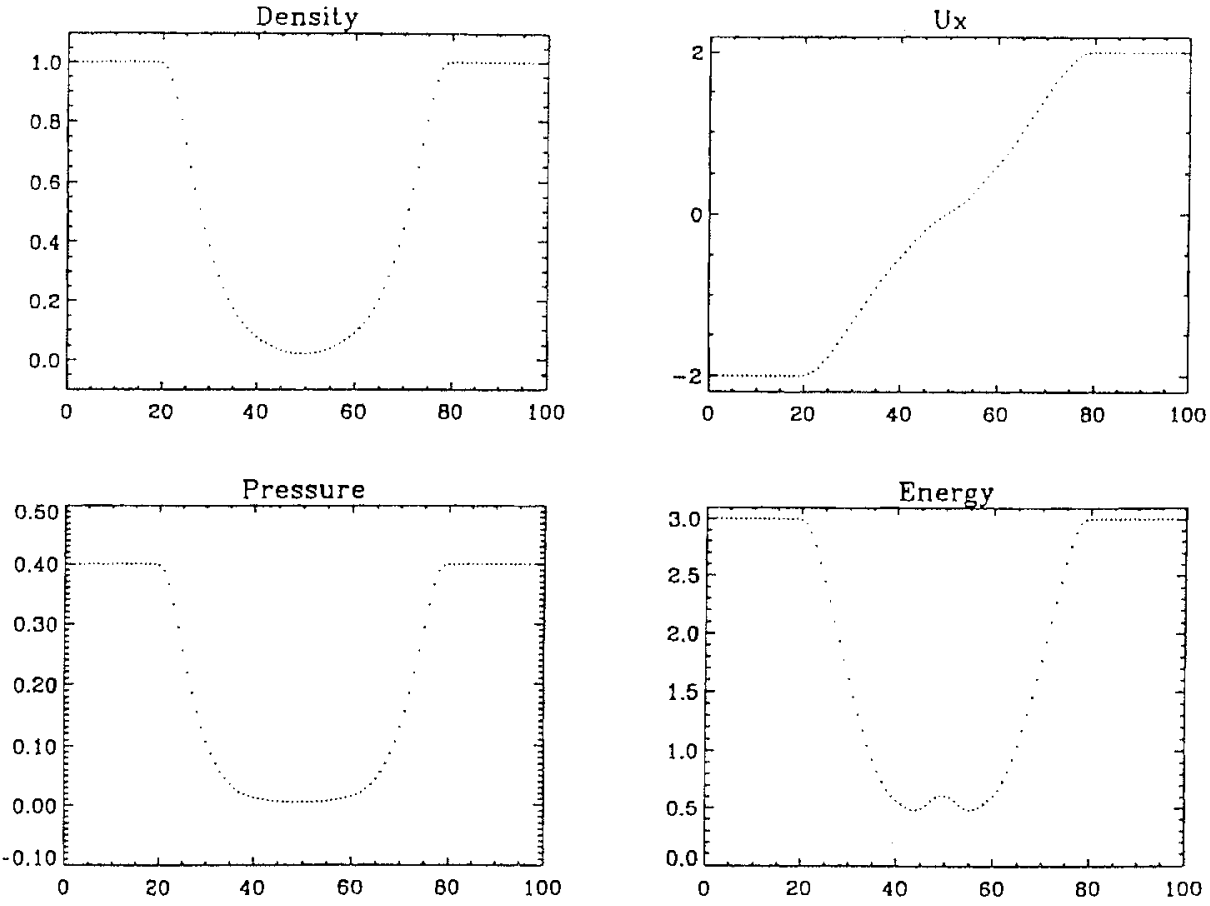

FIG. 5. The solution to the Einfeldt et al. [11] 1-2-0-3 strong rarefaction problem on a mesh with 100 grid points is shown at time 0.1 .

they are very useful tests of the two-dimensional properties of our code. One of the main differences between the purely one-dimensional problems and the two-dimensional problems is that the magnetic field $\mathbf{B}$ varies in the direction of spatial integration in each of the onedimensional sweeps of the operator-split method. This introduces extra source terms in the equations and may give rise to a new set of degeneracies that are not present in the purely one-dimensional problems (see the discussion in the previous section).

3.2.1. Spherical explosion. The explosion is driven by a spherical region (with $r=0.1$ ) with a large overpressure. The initial density and pressure are $\rho=1, P_{e}=1$, and the overpressure is $P_{i}=100$. We have tried three different values of the initially uniform magnetic field: $B_{y}=0, B_{y}=10$, and $B_{y}=100$. Our computation mesh is a cartesian uniform grid with 120 points in each direction. This problem is identical to the one described by Kössl, Müller, and Hillebrandt [14], and we have used their results as a qualitative comparison. In Figs. 6, 7, and 8 , we show the contour plots of density, thermal pressure, magnetic pressure, and kinetic energy for the three cases in which $B_{y}=0, B_{y}=10$, and $B_{y}=100$. For $B_{y}=0$ (Fig. 6), there is one spherically symmetric hydrodynamical shock wave that propagates outward. This solution is essentially identical to the one obtained with other purely hydrodynamical codes, e.g., PPM. For $B_{y}=10$, the shock wave is still mostly spherically symmetric, but it becomes slightly elongated in the direction of the magnetic field.

For $B_{y}=100$, the explosion becomes highly anisotropic: there is essentially no displacement of gas in the direction transverse to the magnetic field, and two hydrodynamical shocks propagate in the parallel direction. In this highly magnetized fluid, the slow magnetosonic wave speed is almost equal to the sound speed, and the fast wave speed is almost equal to the Alfven velocity. Several weak magnetosonic waves are radiated transverse to the magnetic field as an initial transient until total pressure equilibrium is reached at the center. While 

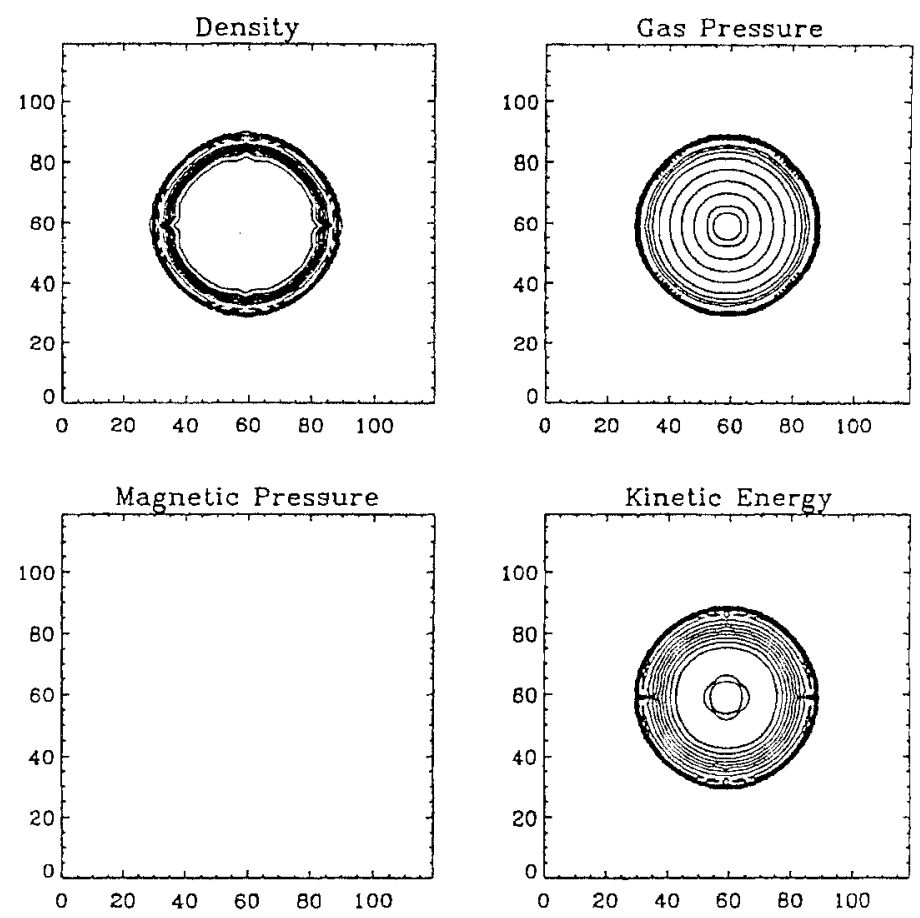

FIG. 6. The solution to the spherically symmetric explosion problem in the hydrodynamic limit $B y_{0}=0$ is shown on a mesh with 120-by-120 grid points after 48 timesteps. For each variable, there are 20 equally spaced contour levels. A CFL condition of 0.8 was used. No artificial viscosity or flattening was applied.
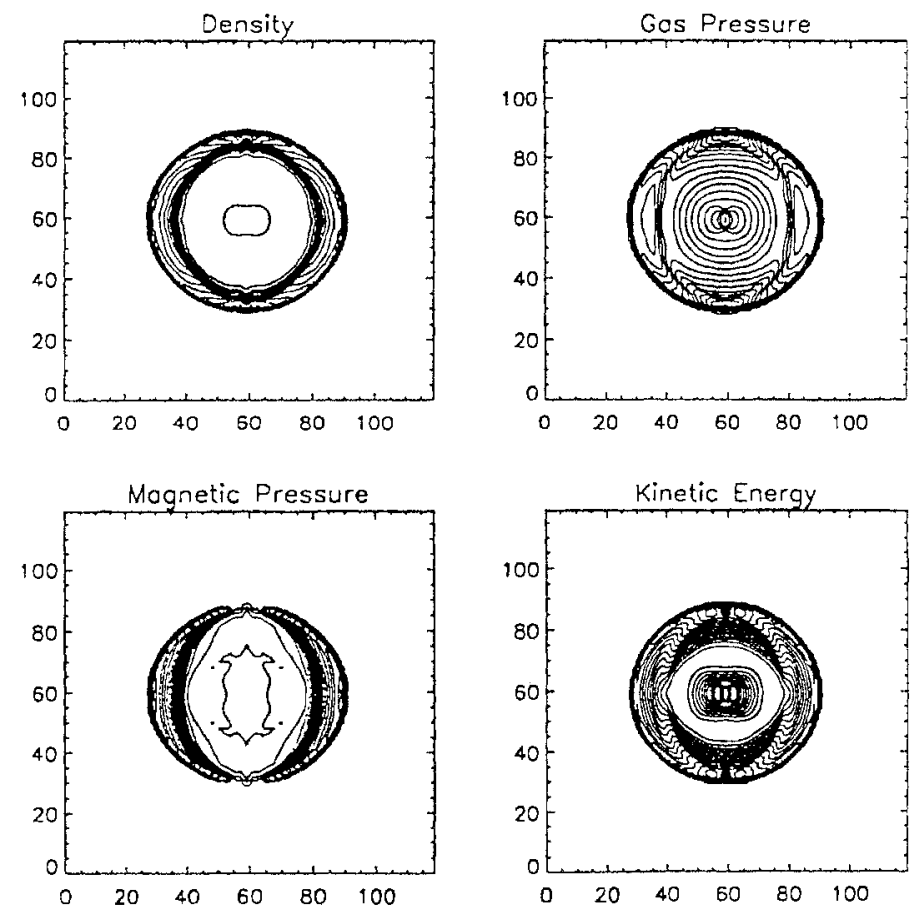

FiG. 7. The solution to the spherically symmetric explosion problem with an initial field $B y_{0}=10$ is shown after 48 timesteps. All the other conditions are the same as in Fig. 6. 

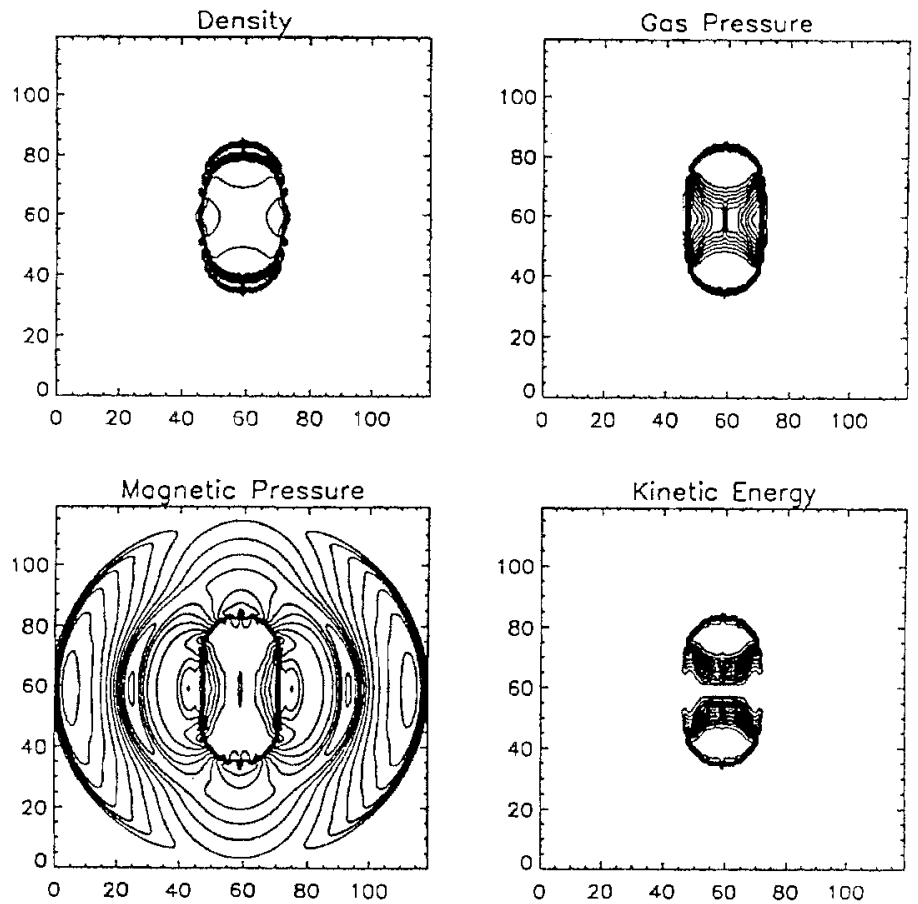

FIG. 8. The solution to the spherically symmetric explosion problem with an initial field $B y_{0}=100$ is shown after 48 timesteps. All the other conditions are the same as in Fig. 6.

the results are in good agreement with the results of [14], we see that the waves and shocks look very sharp and clean with little or no oscillations. The strong steepening of the profiles generates the step-like irregularities that appear in the contour lines near the strong discontinuities. These irregularities are a well-known feature of higher-order Godunov methods (see Woodward and Colella [24]). A flattening algorithm and a quadratic artificial viscosity will cure this effect and prevent the appearance of low-amplitude nonphysical oscillations.

3.2.2. Orszag-Tang vortex. In the second numerical example, we studied the evolution of a compressible Orszag-Tang vortex system like the ones described by Dahlburg and Picone [10], [19]. This problem has been introduced by Orszag and Tang [18] as a simple model to study the evolution of MHD turbulence, and it has been generalized by Dahlburg and Picone [10] for the case of a fully compressible medium. We have selected this problem to test how our two-dimensional code treats the interactions between the several shock waves generated as the vortex system evolves. The initial conditions have a periodic structure containing X-points in both the velocity and the magnetic field. The velocity and magnetic fields have different modal structures. We selected an initially uniform pressure and density based on the value of the average Mach number. The initial configuration is as follows: we choose a cartesian uniform grid with periodic boundaries and 192-by-192 grid points. The fields are given as in [19]:

$$
\begin{aligned}
\rho(x, y, t=0) & =\rho_{0}, \\
P(x, y, t=0) & =P_{0}, \\
\mathbf{v}(x, y, t=0) & =-\sin (y) \hat{\mathbf{x}}+\sin (x) \hat{\mathbf{y}}, \\
\mathbf{B}(x, y, t=0) & =-\sin (y) \hat{\mathbf{x}}+\sin (2 x) \hat{\mathbf{y}},
\end{aligned}
$$



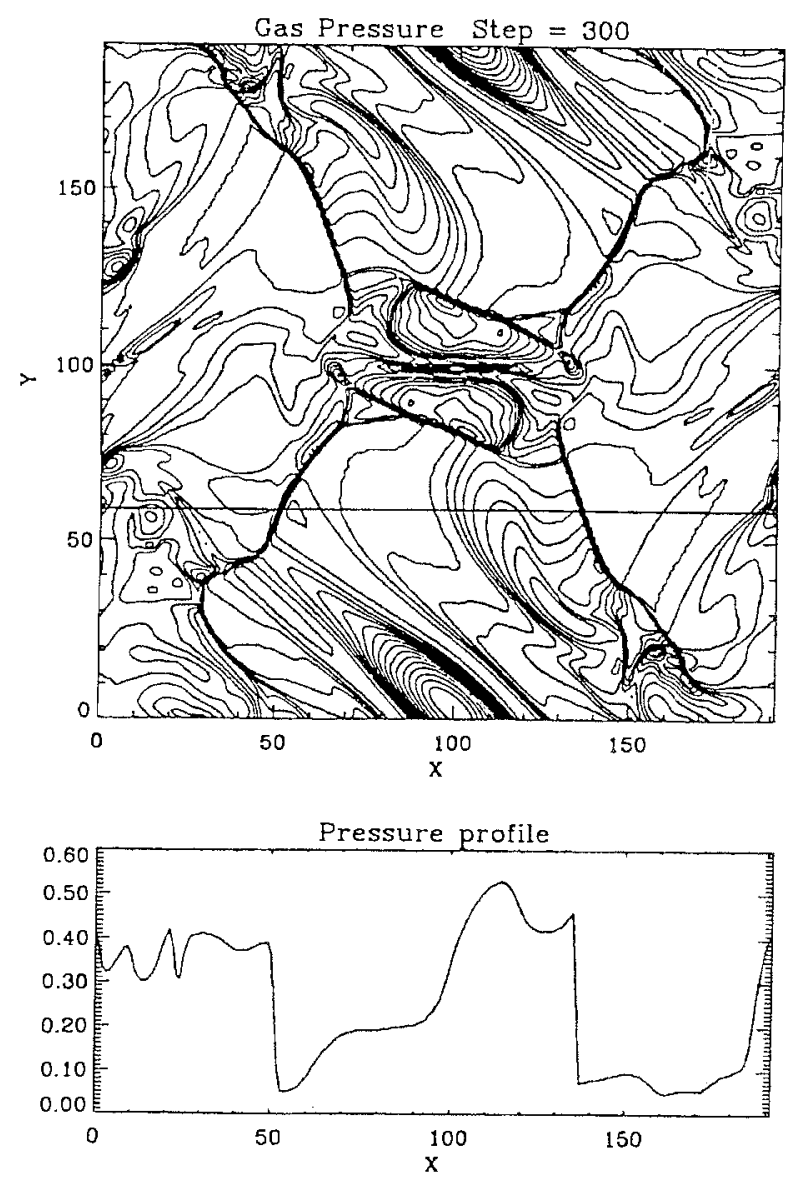

Fig. 9. The evolution of the compressible Orszag-Tang vortex system for the case with initial Mach number $M_{0}=1$ and initial $\beta=\frac{10}{3}$. The solution was computed on a periodic cartesian mesh with 192-by-192 zones and is shown after 300 timesteps (approximately 2 Alfien transit times). The upper plot show's equally spaced contour levels of the thermal pressure. There are several shock fronts that propagate at transverse directions with respect to the grid and eventually interact. The lower plot show's the pressure profile along the solid line at $Y=60$. There are two sharp jumps clearly visible where the solid line intersects the wo strong shock fronts.

where $\hat{\mathbf{x}}$ and $\hat{\mathbf{y}}$ are unit vectors in the $x$ and $y$ directions. The initial average Mach number is given by $M^{2}=\rho_{0}\left|\mathbf{V}_{0}\right| /\left(\gamma P_{0}\right)$, where $|\mathbf{V}|_{0}$ is the initial root mean square (rms) value of the velocity. The initial average $\beta$, i.e., the ratio of thermal and magnetic pressure, is $\beta=8 \pi P_{0}\left|\mathbf{B}^{2}\right|$.

We examined the case with $M=1.0$ and $\beta=\frac{10}{3}$. In Fig. 9 we present the contour profiles of the thermal pressure after 300 timesteps. The plot shows the pressure profile along the solid line at $y=60$, and it shows how the shocks remain sharply defined within a few grid points even in two dimensions. There are several shock fronts that propagate at different angles with respect to the grid, and which interact until the vortex system decays and gives rise to small-scale structures. The dynamics of the decay appears to closely match the one described in Dahlburg and Picone [10], even though we are not using exactly the same initial conditions. We note that in our code, the decay of the vortex system is determined by the numerical viscosity and resistivity that is built into the dissipation mechanism of the code. The similarity between our results and the results of Dahlburg and Picone, who use physical dissipation, therefore suggests that the numerical dissipation indeed provides a good model for the physical diffusive processes at subgrid scales. This conjecture, which deserves further 
experiments, has been advanced also for the case of hydrodynamic turbulence by, for example, Cattaneo et al. [6].

4. Discussion and conclusions. We have derived an explicit, second-order Godunov method for the equations of ideal MHD in two and three spatial dimensions. Our approach contains many of the same elements applied successfully to ideal, compressible hydrodynamics. Most of the general techniques that improve the behavior of Godunov methods in hydrodynamics, such as flattening and artificial viscosity, carry over to our code. Our main innovation is the construction of an approximate, linearized Riemann solver. This Riemann solver detects and handles the degeneracies that occur in ideal MHD, and is well behaved for physically acceptable states. By well behaved we mean that the Riemann solver does not produce nonphysical states like, for example, negative pressures and densities in the presence of strong rarefaction waves.

To apply the one-dimensional characteristic analysis to the formulation of the Riemann problem. we rewrote the equations in quasi-conservative form. That is, we handle the terms containing derivatives of the magnetic field component parallel to each spatial direction, $\partial B_{x} / \partial x$ and $\partial B_{y} / \partial y$, as source terms, not as fluxes. In general coordinates, we split the terms in the equations into different components: some that must be treated as divergences, some as gradients, and some as pure source terms. Our particular quasi-conservative formulation is well suited to the operator-split methodology on which we based our code. At each timestep, we solve two sets of one-dimensional equations, one set for each spatial direction. These equations are not the same as the purely one-dimensional equations because they contain source terms arising from the Maxwell equation $\nabla \cdot \mathbf{B}=0$. Furthermore, we wrote the momentum equations in such a way that the condition $\nabla \cdot \mathbf{B}=0$ applies exactly to the continuum equations, even if it does not apply to the discretized version. For the examples we have considered, our approach appears to suppress the occurrence of monopole forces that would lead to unphysical dynamics. In cases where our basic difference approximation does not suffice to maintain the divergence-free condition of the magnetic field, we have the option of applying a Hodge decomposition to the magnetic field at each timestep to eliminate the monopole component.

The code achieves high resolution and accuracy in the presence of strongly discontinuous solutions. This high performance has some limitations, however. Because performing a Riemann solve is computationally expensive, and because the time advance of the solution is limited by the CFL condition, our code is best suited to studying transient phenomena that involve the propagation and interaction of shock discontinuities and MHD waves. While the current formulation of the code can already be used to study a wide variety of problems, there are good reasons to construct a fully unsplit Godunoy scheme for the MHD equations. We expect that an unsplit method will be more appropriate when studying systems with strongly sheared fields, and that generally such an approach will improve the advection of the solenoidal magnetic field. This problem is analogous to the advection of the velocity field in an incompressible medium. Colella [8] discusses a general method of constructing unsplit methods that make use of the multidimensional wave propagation properties of the solution to construct the Godunov fluxes. We believe that the same approach will work also for the equations of MHD, and that our Riemann solver will be the base of one such scheme. As usual, the final answer must rely on numerical experiments.

Acknowledgments. Andrea Malagoli wishes to thank D. Spicer and the staff of the NASA Center for Computational Sciences (NCCS) for providing the supercomputing resources. We also thank Cray Research for generously allowing us access to its computer resources. 


\section{REFERENCES}

[1] J. B. BELL, P. COLELla, AND H. M. GLAZ, A second-order projection method for the incompressible Navier-Stokes equations, J. Comput. Phys., 85 (1989). pp. 257-283.

[2] J. B. BeLl, P. COLELLA, AND L. Howell, An efficient second-order projection method for viscous incompressible fiou, Proc. 10th AIAA Computational Fluid Dynamics Conf., Honolulu, HI, June 24-27, 1991, pp. 360 367.

[3] J. B. BELL. P. COLELLA, AND J. A. TRAGENSTEIN. Higher order Godunov methods for general systems of hyperbolic conservation laws, J. Comput. Phys., 82 (1989), pp. 362-397.

[4] J. U. BRACKBILL AND D. C. BARNES, The effect of nonzero $\nabla \mathbf{B}$ on the numerical solution of the magnetohydrodynamic equations, J. Comput. Phys., 35 (1980), pp. 426-430.

[5] M. BRIO AND C. C. WU, An upwind differencing scheme for the equations of ideal magnetohydrodynamics, J. Comput. Phys.. 75 (1988), pp. 400-422.

[6] F. Cattaneo, N. H. Brummell, J. Toomre, A. Malagoli, and N. E. Hurlburt, Turbulent compressible convection, Astrophys. J., 370 (1991), pp. 282-294.

[7] P. COLELLA, A direct Eulerian MUSCL scheme for gas dynamics, SIAM J. Sci. Statist. Comput., 6 (1985), pp. 104-117.

[8] - Multidimensional upwind methods for hyperbolic conservation laws, J. Comput. Phys, 87 (1990), pp. 171-200.

[9] P. Colella AND P. R. Woodward, The piecewise parabolic method (PPM) for gas-dynamical simulations. J. Comput. Phys., 54 (1984), pp. 174-201.

[10] R. B. DahlBurg AND J. M. Picone, Evolution of the Orszag-Tang vortex system in a compressible medium. I. Initial average subsonic flow, Phys. of Fluids B, 1 (1989), pp. 2153-2171.

[11] B. Einfeldt, C. D. Munz. P. L. RoE, AND B. SJögreen, On Godunov-type methods near low densities, J. Comput. Phys., 92 (1991), pp. 273-295.

[12] B. ENGQUIST AND S. OSHER, One-sided difference approximations for nonlinear conservation laws, Math. Comp., 36 (1981), pp. 321-351.

[13] A. HARTEN, P. D. LAX, AND B. van LeER, On upstream differencing and Godunov-type schemes for hyperbolic conservations law's. SIAM Rev., 25 (1983), pp. 35-61.

[14] D. Kösst, E. MÜller, AND H. HILlebrandT, Numerical simulations of axially symmetric magnetized jets: I. The influence of equipartition magnetic fields, Astron. and Astrophys., 229 (1990), pp. 378-396.

[15] P. D. LAX, Weak solutions of nonlinear hyperbolic equations and their numerical computation, Comm. Pure Appl. Math. 7 (1954), pp. 159-193.

[16] - Hyperbolic systems of conservation laws and the mathematical theory of shock waves, Conference Board of the Mathematical Sciences Regional Conference Series in Applied Mathematics, Society for Industrial and Applied Mathematics. Philadelphia, PA, 1973.

[17] T. P. LiU, The Riemann problem for general systems of conservation laws, J. Differential Equations, 18 (1981), pp. 218-234.

[18] S. A. ORSZAG AND C. M. TANG, Small-scale structure of two-dimensional magnetohydrodynamic turbulence, J. Fluid. Mech., 90 (1979), pp. 129-143.

[19] J. M. PTCONE AND R. B. DAHLBURG, Evolution of the Orszag-Tang vortex system in a compressible medium. II Supersonic flow, Phys. of Fluids B, 3 (1991), pp. 29-44.

[20] P. L. ROE, Approximate Riemann solvers, parameter vectors, and difference schemes, J. Comput. Phys., 53 (1981), pp. 357-372.

[21] V. V. Rusanov, Calculation of interaction of non-steady shock waves with obstacles, Zh. Vychisl. Mat. i Mat. Fiz., 1 (1961), pp. 267-279.

[22] G. SoD, A survey of several finite difference methods for systems of nonlinear hyperbolic conservation law's, J. Comput. Phys., 27 (1978), pp. 1-31.

[23] B. VAN LEER, TOwards the ultimate conseriative difference scheme IV. A new approach to numerical convection, J. Comput. Phys., 23 (1977), pp. 276-299.

[24] P. R. WOODWARD AND P. COLELLA, The numerical simulation of two-dimensional fluid flow with strong shocks, J. Comput. Phys., 54 (1984), pp. 115-173.

[25] A. ZACHARY AND P. COLELLA, A higher-order Godunov method for the equations of ideal magnetohydrodynamics, J. Comput. Phys.. 99 (1992). pp. 341-347. 